\title{
Building a New Software of Electromagnetic Lenses (CADTEL)
}

\author{
Hussain S. Hasan \\ Department of Physiology and Medical Physics, College of Medicine, \\ Al-Nahrain University, Baghdad, Iraq. \\ E-mail address: altai1965@yahoo.com
}

\begin{abstract}
The Computer Aided designing Tools for Electromagnetic Lenses (CADTEL) is a new software as application package of programs concept to aid both users and professionals in symmetric and asymmetric electromagnetic lenses with single, double, and multipole piece in electron microscope of electron optics field. The CADTEL software has been designed to run on several computer platforms and includes two types of design procedure. The first one named, analysis procedure (which is based on trial and error) where consist of three sorts called; $\mathrm{H} 1$ programs for magnetic scalar potential by solving Laplace's equation [1,2], $\mathrm{H} 2$ and $\mathrm{H} 3$ programs for magnetic vector potential by solving Poisson's equation $[3,4]$ in linear and non linear media respectively. While the second part, named $\mathrm{H} 4$ programs for the synthesis procedure (inverse design). The previous types differ by the obtaining the magnetic flux density, and poles shape, while it is analog by compute and plot the lens properties which are operating at zero, low, high, and infinite magnification conditions (operation modes). CADTEL software consist of computational and plot steps of magnetic field, equipotential surfaces, flux lines, objective and projector properties, and poles shape for proposed lens design which are appear in auto visual interfaces, that are coded in visual basic language [5].
\end{abstract}

Keywords: Electron lenses; Objective and projector properties; Lenses aberrations; Electron optics; Charged particle optics; Software and simulation in electron lenses

\section{INTRODUCTION}

From literature survey, there are numerous of software and programming packages or simulation have been presented for example; SPOC software [6], G-Optk simulation software [7], EOD program [8], CPO programs [9], Focus software [10], SIMION software [11], and [12], EOS Simulator [13], MEBS software [14], OmniTrak software [15]. For computation and comparison, the one can use Munro's lenses [16] as proposed lenses and supply the lens data to current software in analysis procedure. In the synthesis procedure, the user can apply any mathematical model which he wanted as a ready magnetic field model or choosing one of the fifteen models which are introduced in the software such as Glaser model [17], GrivetLenz model [18], Gaussian model [18], Spherical model [19], Exponential model [19],...etc. Electromagnetic lenses programs codes play a role in the design process and enhanced software tools offer the promise of improved productivity for lenses designers. Another benefit of improved software tools a significant reduction in the time required to train new researchers (users) in the use of electromagnetic lens simulation programs. In addition to reduce the potential and tedious. This paper summarizes operating such new tool named CADTEL with ability to make the many developments and improvements nearly. 


\section{THEORY}

Electron lens is an instrument play an important rule for controlling beams of electrons concerning the acceleration, focusing and deflecting. This lens suffer from several aberrations such as spherical and chromatic aberration when uses as objective lenses [20], and distortions such as radial and spiral distortion when uses as projector lenses [21, 22, 23]. The minimum values of aberrations and distortions leads to the optimization.

\section{VISUAL INTERFACES}

Each program of CADTEL software has a visual form displayed on the computer screen (by choosing the option which is the user select from the main page of software) named the home page contains mainly the menus bar consist of several commands as shown in figure 1 , some of images sense with lens subject, picture boxes as environment of graphs, and text boxes for titles and input data as shown in figure 2, and figure 3.

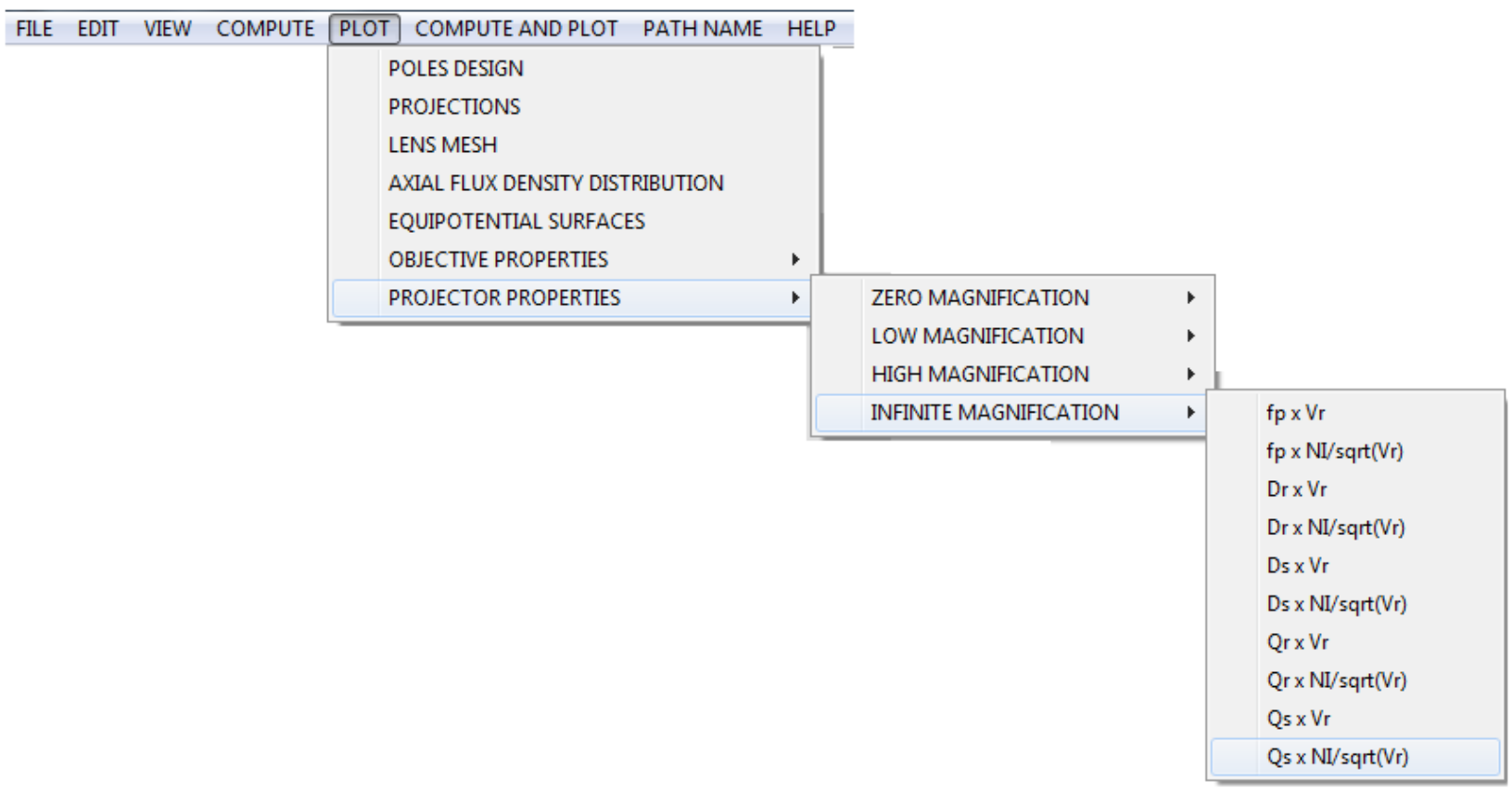

Fig. 1. Sample of command and sub-command options of H1 program menu bar for symmetric and asymmetric lenses in executive stage for CADTEL software. 
FILE EDIT VIEW COMPUTE PLOT COMPUTE AND PLOT PATH NAME HELP

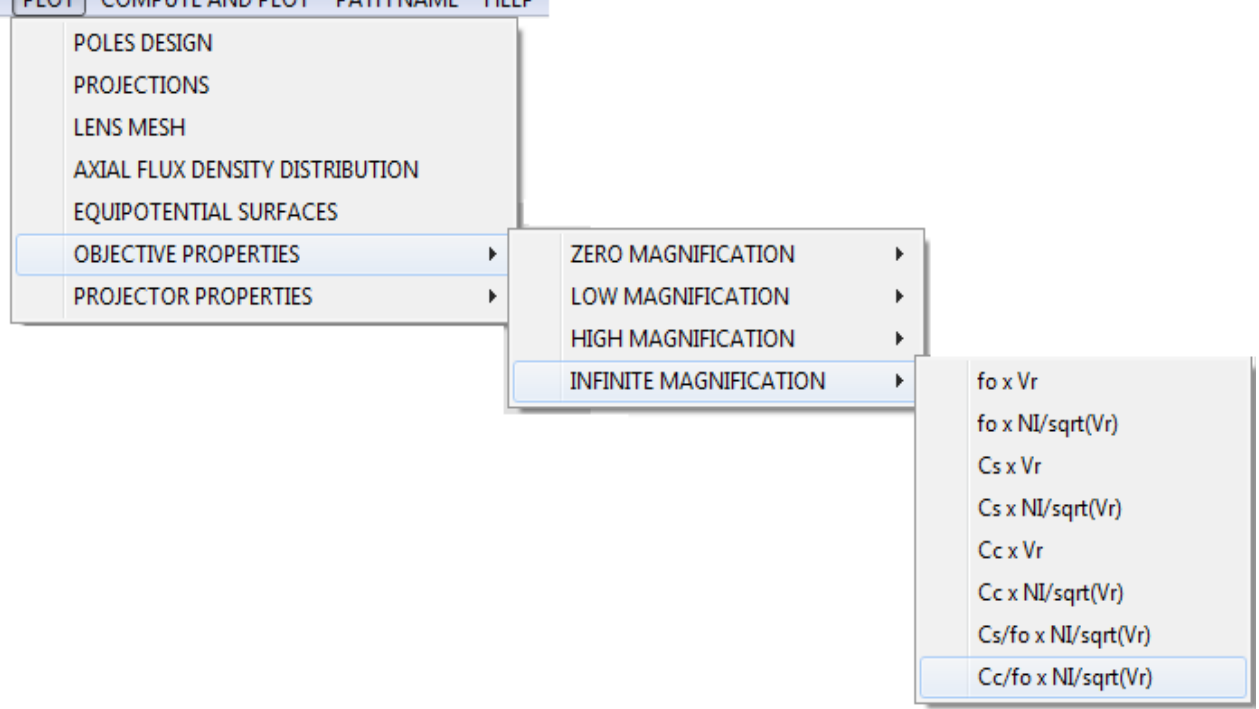

Fig. 1 (continuation). Sample of command and sub-command options of H1 program menu bar for symmetric and asymmetric lenses in executive stage for CADTEL software.

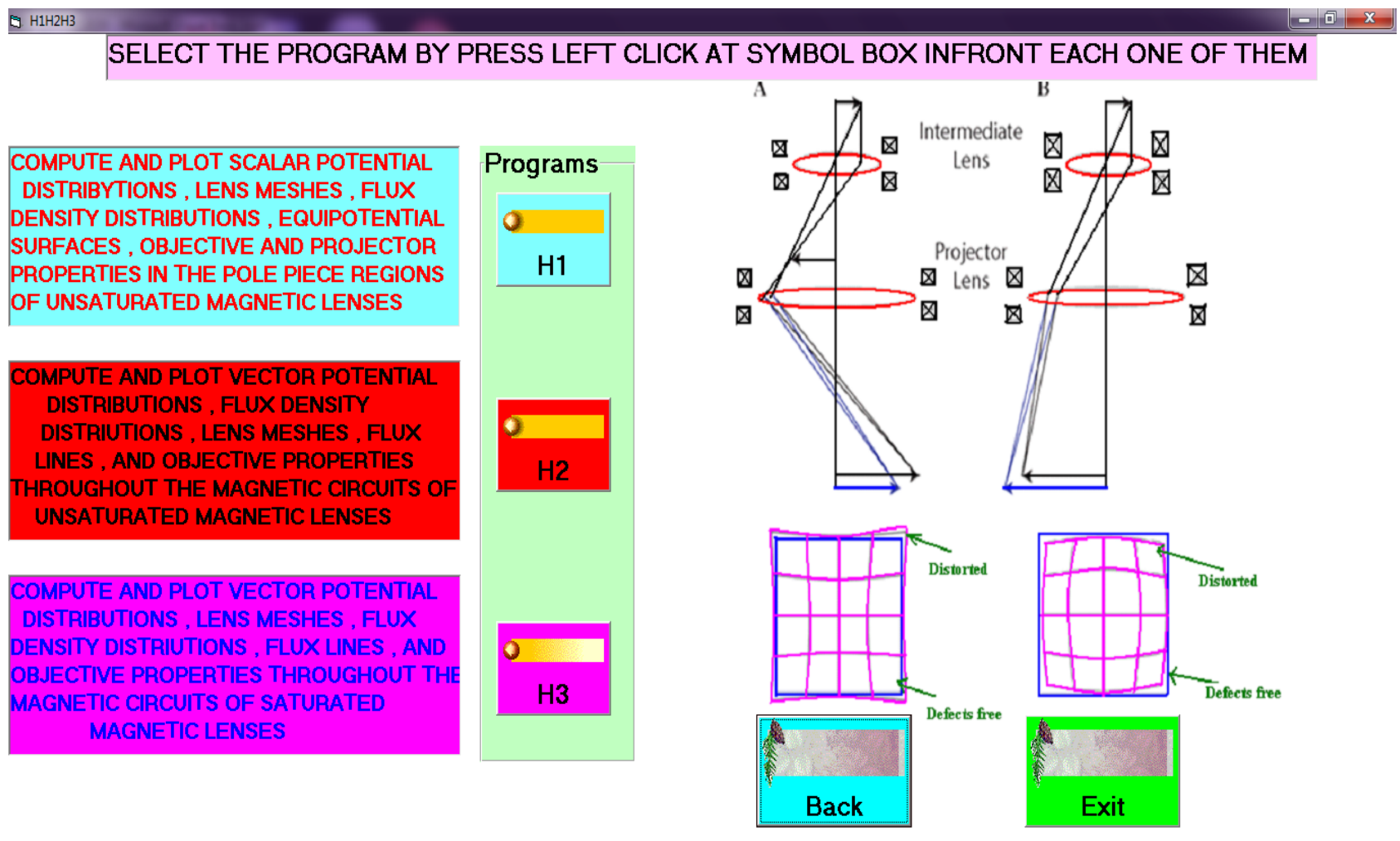

\section{(4) $\mathrm{D}$ (3) $\mathrm{B}$ e 0 :}

Fig. 2. The page of analysis procedure of CADTEL. 

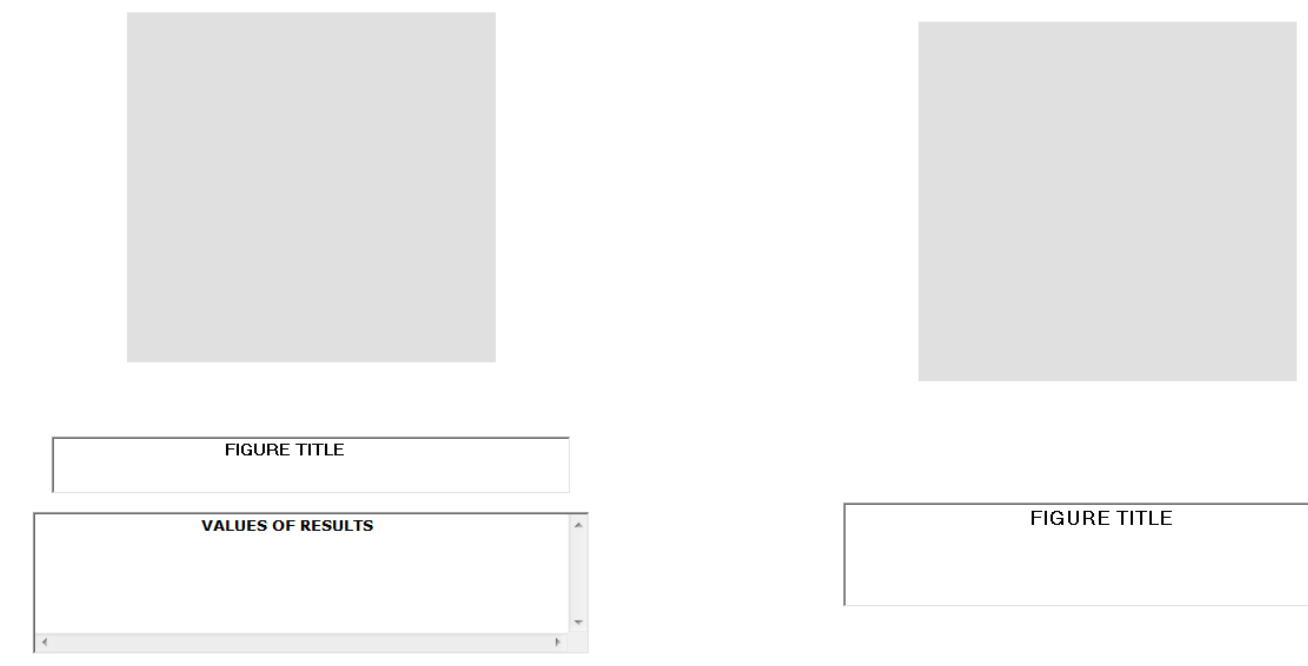

FIGURE TITLE

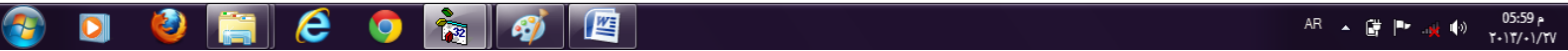

Fig. 3. The home page of asymmetric lens in $\mathrm{H} 2$ program.

\section{EDITING INPUT DATA AND OUTPUT RESULTS}

One of emphasis in the CADTEL is on assisting users in the setup and running of the electromagnetic lens software without requiring any knowledge of the format, syntax or similar requirements of the input data and output results. Automatically, the input data setup in external file at certain path, while received the output results in text boxes directly at the home page of each program in addition to external file at certain path which is also appear in executing stage. This results provide users with further useful feedback on their proposed lens in addition to auto use it as input data of plot option. The user may select different options, are immediately displayed for compute and plot or any command in FILE, EDIT, and VIEW options as one of functions may be the user need it.

\section{RESULTS AND DISCUSSION \SELECTING OUTPUT DISPLAYS}

Several run time output display options are available by using the introduced interfaces. Figure 4 represent the proposed mesh grid of electromagnetic symmetric lens by using H1 program. While figure 5 shows the magnetic flux lines of electromagnetic asymmetric lens by using H2 program. Sample of axial magnetic flux density distribution for electromagnetic symmetric lens by using $\mathrm{H} 3$ program is shown in figure 6 . Figure 7 refer to axial flux density and magnetic scalar potential according to Exponential model. Thus, the reconstructed profile pole piece depending on Glaser model by using H4 program is shown in figure 8 . Specimen of properties for proposed lens are obtained from the CADTEL programs, shows in figure 9 for spherical aberration $C_{s}$, figure 10 for radial distortion $D_{r}$, and figure 11 for projector focal length $f_{p}$. The visual interfaces of CADTEL software provides an automated procedure for doing the above commands such as computations and scatter plots, and other functions may also be displayed during a run. 


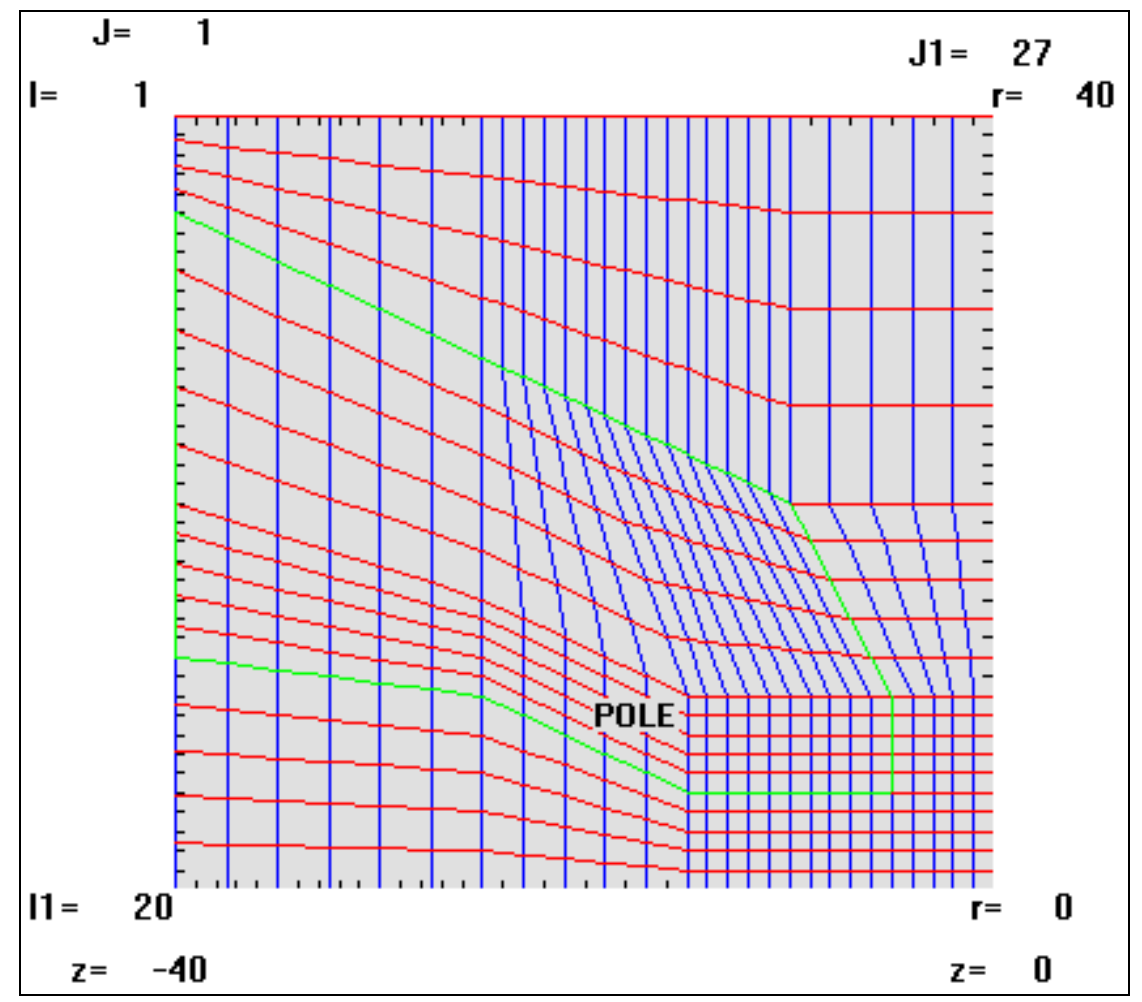

Fig. 4. The produced mesh grid for symmetric lens.

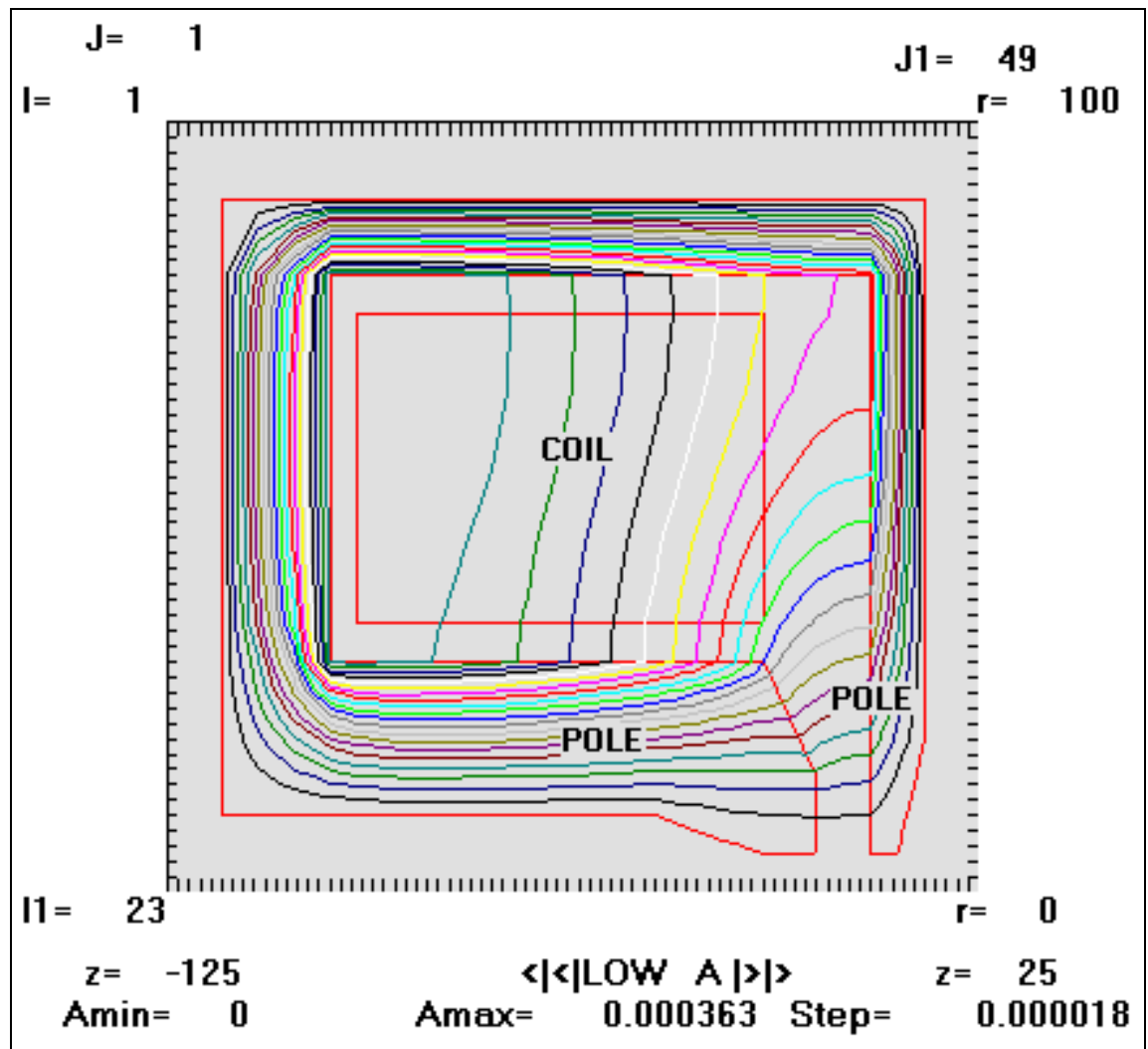

Fig. 5. The plot of the computed magnetic flux lines throughout the iron circuit and coil windings for Munro's asymmetric lens. 


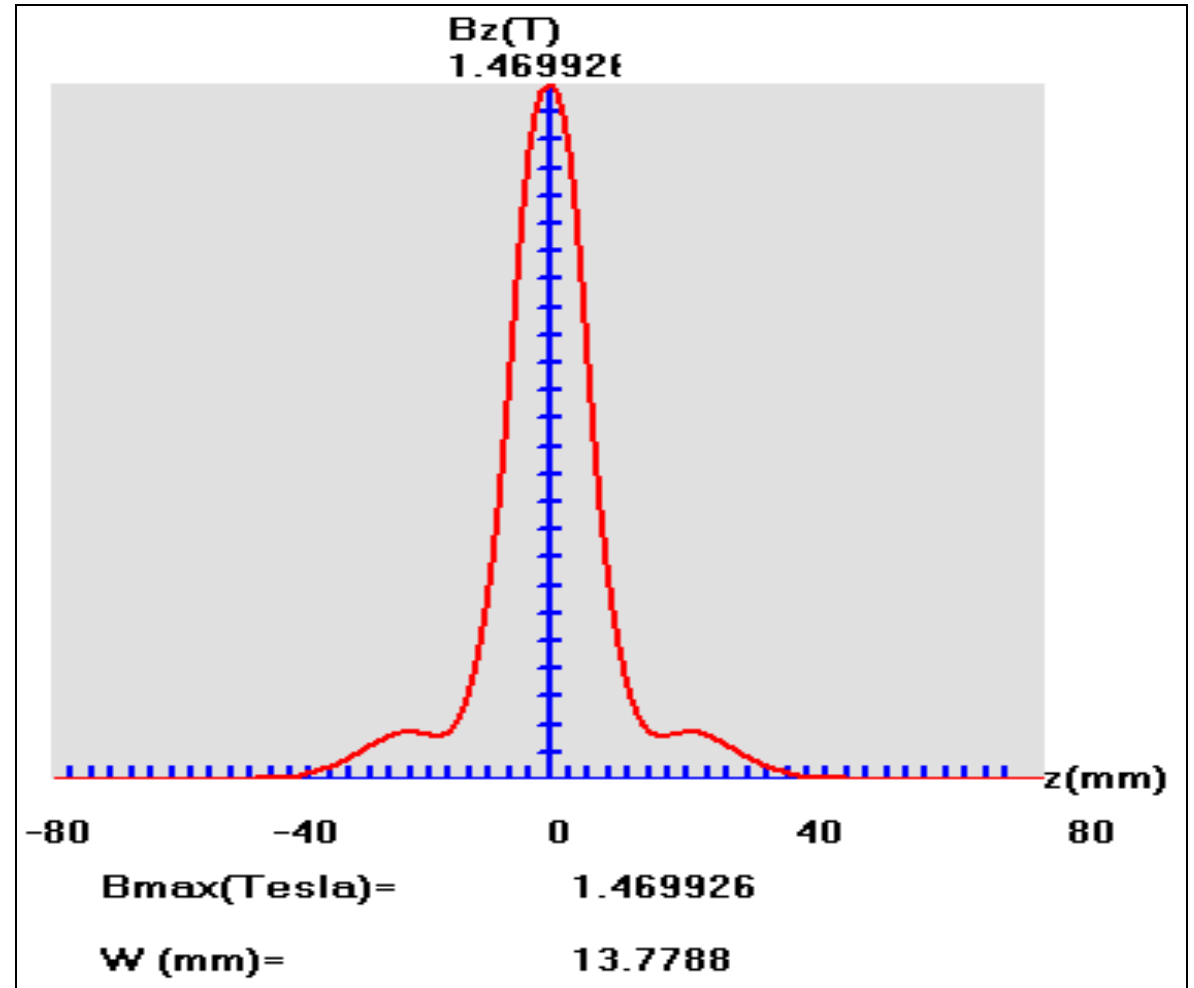

Fig. 6. The axial flux density distribution as a function of axial distance $\mathrm{z}$ for Munro's symmetric lens.

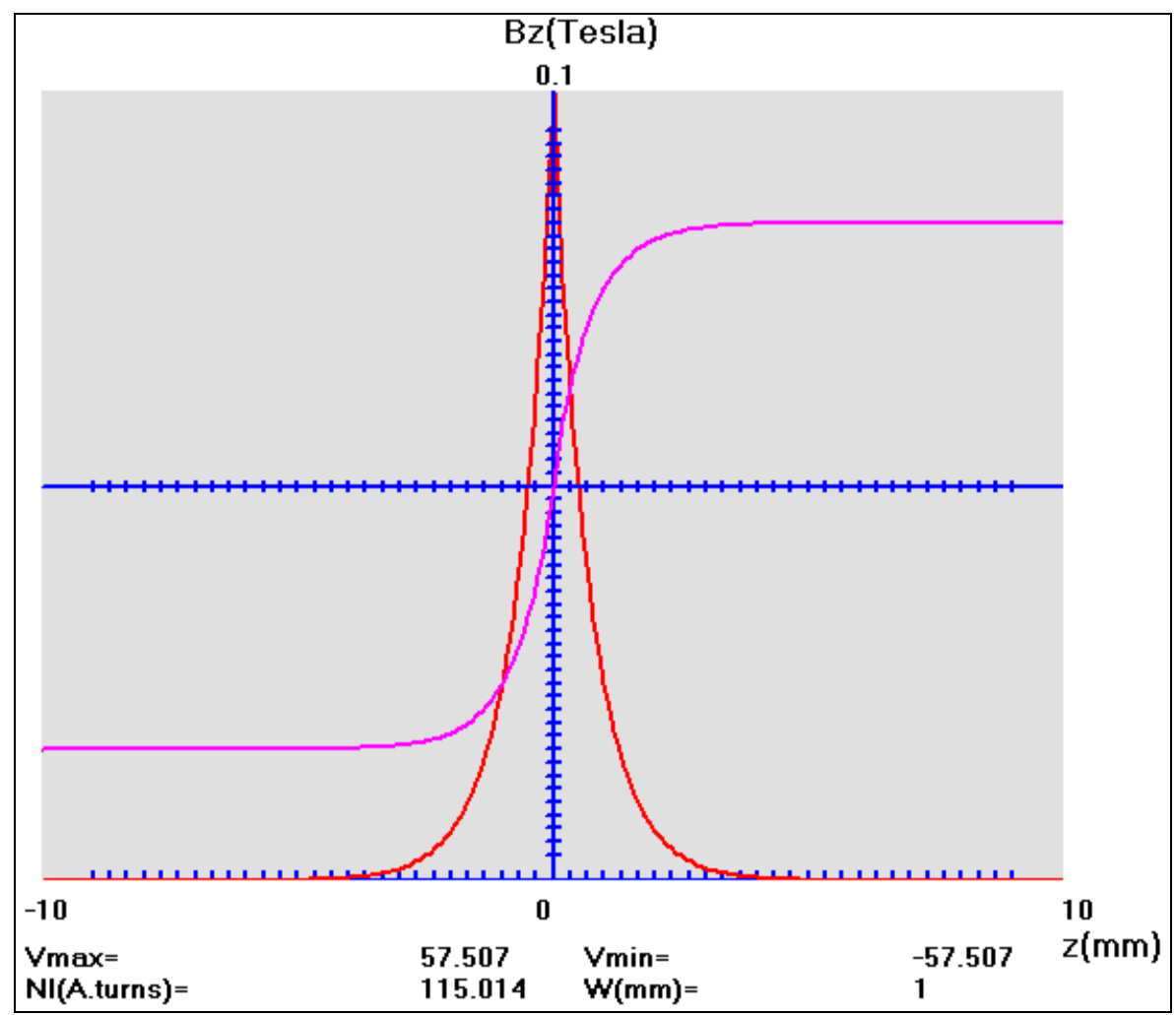

Fig. 7. The axial flux density distributions and scalar magnetic potential for symmetric proposed lens as a function of axial distance for Exponential model. 


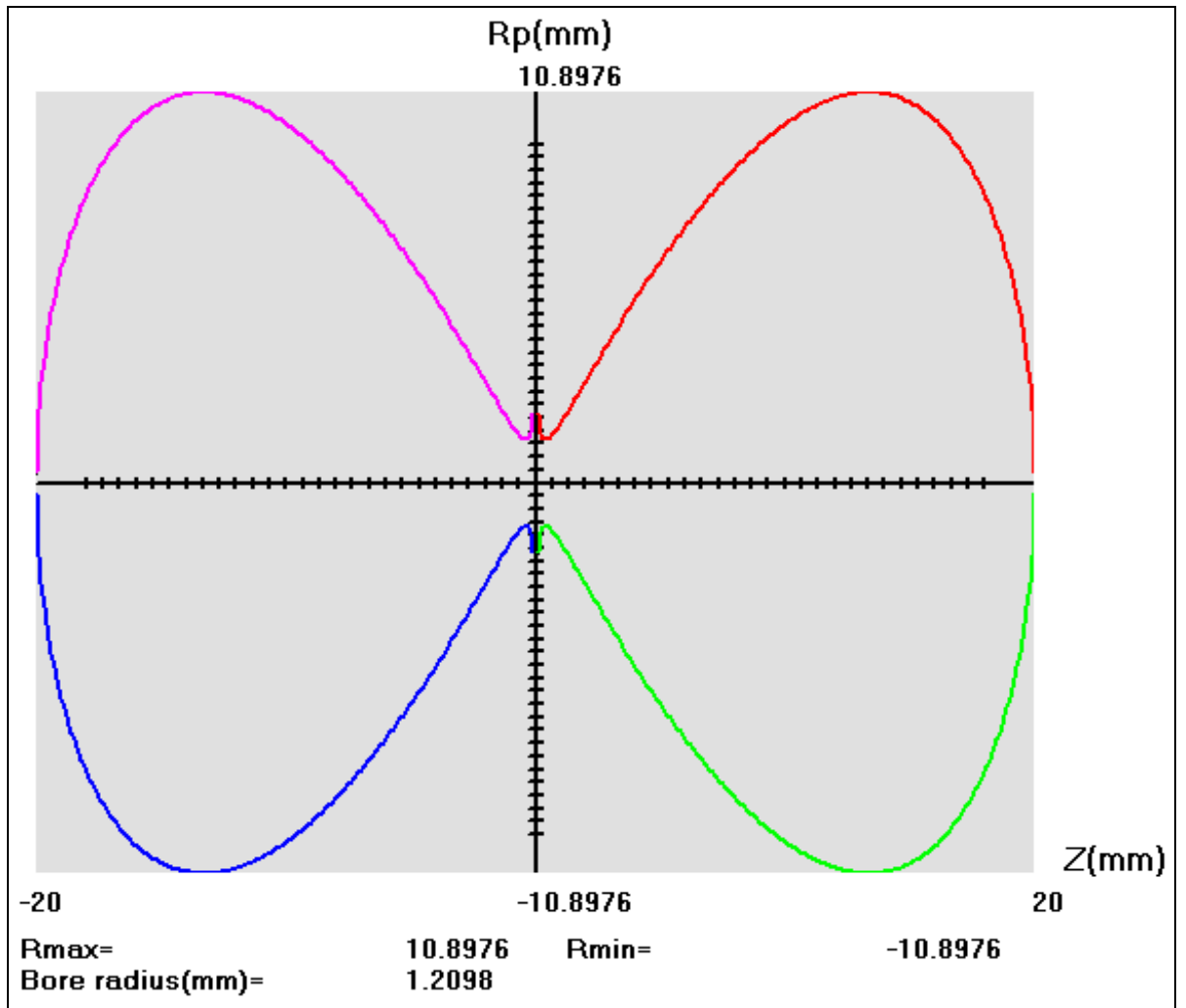

Fig. 8. The polepiece profile reconstruction for symmetric proposed lens as a function of axial distance for Glaser model.

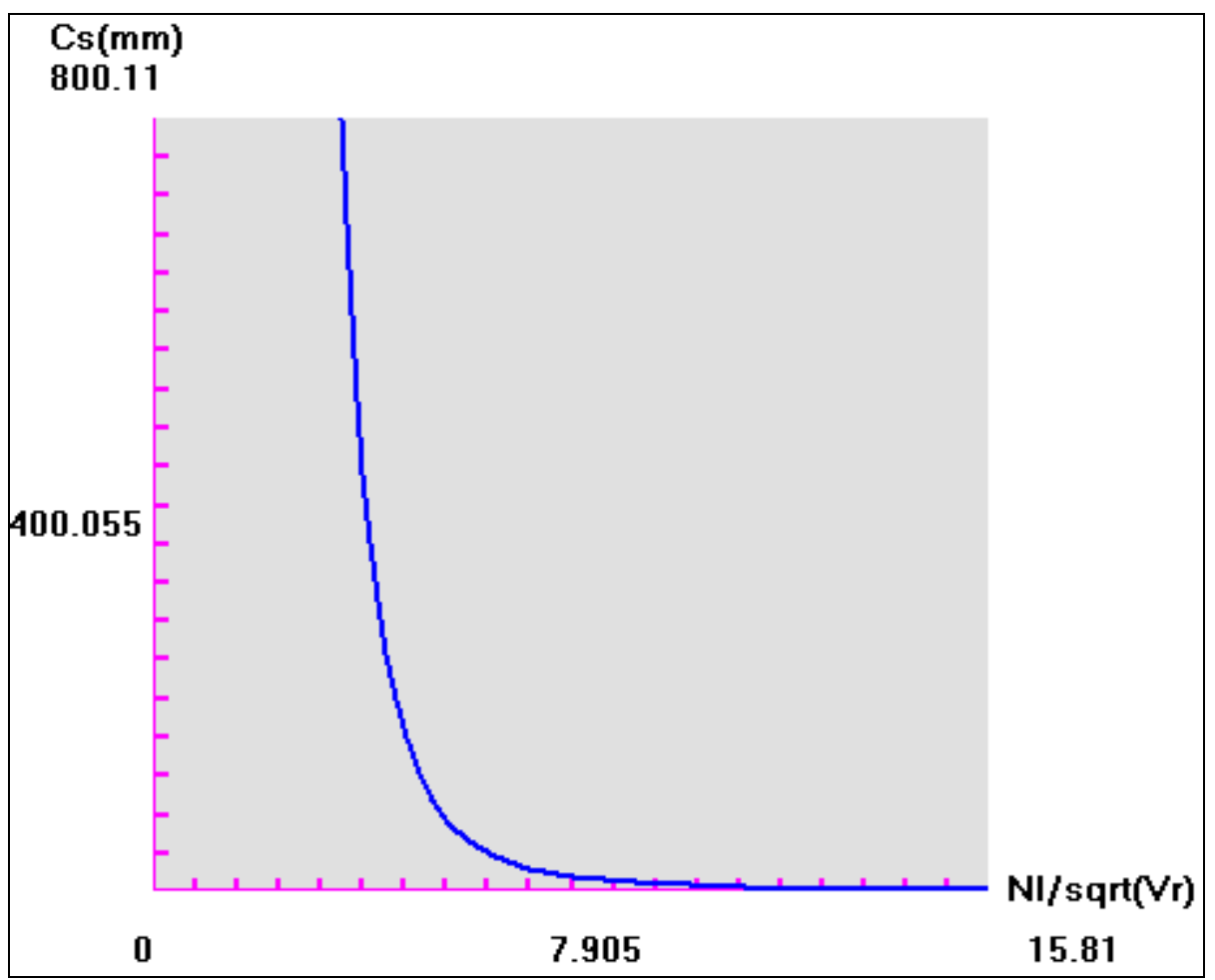

Fig. 9. The spherical aberration $C_{s}$ as a function of excitation parameter $\mathbf{N I} / \sqrt{\mathbf{V r}}$ for asymmetric proposed lens at zero magnification condition by using H1 program. 


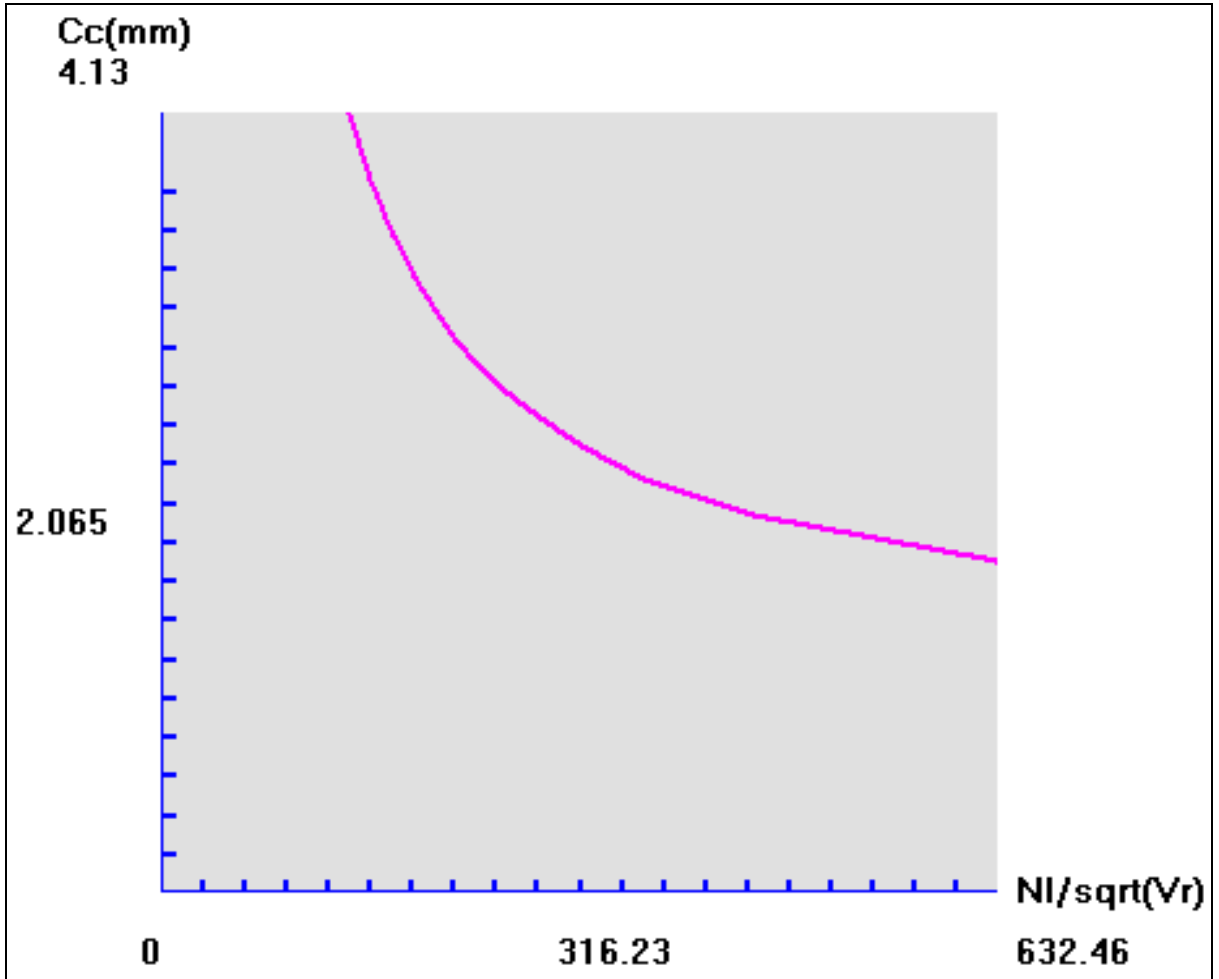

Fig. 10. The chromatic aberration $C_{c}$ as a function of excitation parameter $\mathbf{N I} / \sqrt{\mathbf{V r}}$ for asymmetric proposed lens at zero magnification condition by using $\mathrm{H} 3$ program.

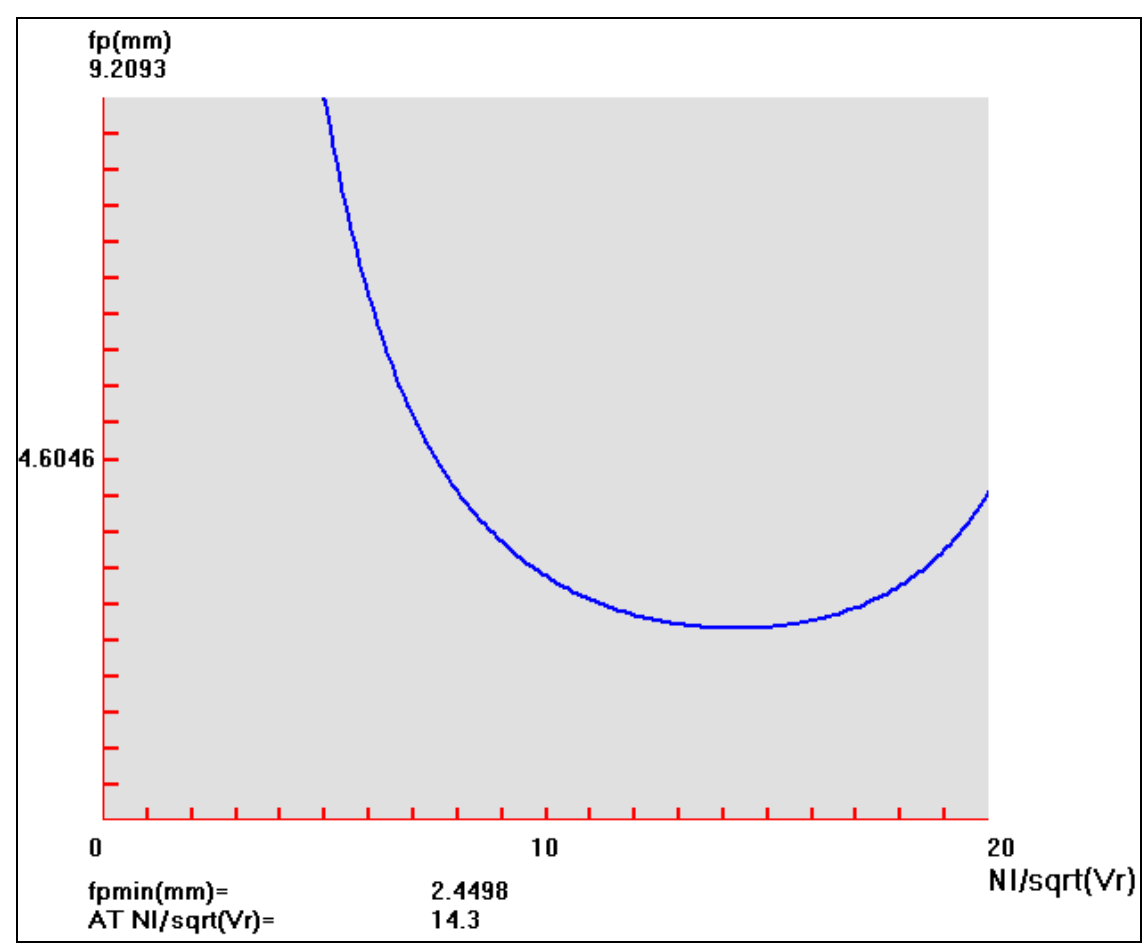

Fig. 11. The projector focal length $f_{p}$ as a function of excitation parameter $\mathbf{N I} / \sqrt{\mathbf{V r}}$ of symmetric proposed lens for Three Halves Exponent model at infinite magnification condition by using H4 program. 


\section{CONCLUSION}

CADTEL software is a useful new tool for electromagnetic lenses design and analysis. This software provides an interactive and intuitive package for designing symmetric and asymmetric lenses for more than pole piece and for four operation modes in both, the analysis and synthesis procedure. CADTEL is a significant reduction in the time and potential required to train new researchers in the use of electron optics programs. So, both experience and new users of electron optics field should realize increased productivity.

\section{ACKNOWLEDGEMENT}

The author is indebted to Hassan N. Al-Obaidi, Al-Mustansiriyah University, for constructive comments. Thus, Eric Munro, University of Cambridge, due to using his lenses as proposed lens to test current software.

\section{References}

[1] H. H. Rose, Geometrical Charged-Particle Optics, Springer Series in Optical Sciences, 2009.

[2] S. I. Molokovsky, Intense Electron and Ion Beams, Springer-Verlag Berlin Heidelberg, 2005.

[3] A. Zhigarev, Electron Optics and Electron Beam-Devices, (Mir. Publisher: Moscow), 1975.

[4] M. Reiser, Theory and Design of Charged Particle Beams, WILEY-VCH Verlag $\mathrm{GmbH} \& \mathrm{Co} . \mathrm{KGaA}$, Weinheim, 2004.

[5] E. Petroutsos, Mastering ${ }^{T M}$ Visual Basic ${ }^{\circledR}$ 6, (C) SYBEX Inc. U.S.A, 1998.

[6] B. Lencova, Software for Particle Optics Computations SPOC, Fleischnerova 15, 63500 Brno, Czech Republic, 2000.

[7] Sh. Fujita, M. Takebe, H. Shimoyama, G-Optk: Simulation Software for Electron Gun Characterization", Seventh International Conference on Charged Particle Optics (CPO-7), Abstract for Computer Software Demonstrations, Trinity College Junior Parlour, 2006.

[8] B. Lencová, J. Zlámal, Microscopy and Microanalysis 13 (3) (2007) 2-3.

[9] H. Müller, S. Uhlemann, P. Hartel, M. Haider, "Aberration-Corrected Optics: From an idea to a device", Proceeding of CPO-7, Eds. E. Munro and J. Rose, Physics Procedia 1(1) (2008) 167-178.

[10] A. A. Trubitsyn, Applied Physics (Rus.) 2 (2008) 56-62.

[11] D. A. Dahl, Int. J. Mass Spectrom. 200 (2000) 3-25.

[12] D. Manura, "SIMION ® 8.0 User Manual", Scientific Instrument Service (SIS), Inc., issue 172, USA, 2008.

[13] T. Huang, Q. Hu, Z. Yang, B. Li, J. Q. Li, X. L. Jin, Y. L. Hu, X. F. Zhu, L. Liao, L. Xiao, G. X. He, Electron Devices, IEEE Transactions on 56(1) (2009) 140-148. 
[14] E. Munro, Munro's Electron Beam Software - Software Catalogue, MEBS Ltd, 2010.

[15] S. Humphries, Three-dimensional Charged-particle Optics and Gun Design, Field Precision LLC, CRC Press, Albuquerque, New Mexico U.S.A, 2011.

[16] E. Munro, A Set of Computer Programs for Calculating of Properties Electron Lenses, Univ. of Cambridge, Depart. Of Eng. Report CUED/B- Elect. TR 45, 1975.

[17] R. F. Egerton, Physical Principles of Electron Microscopy, Springer, USA, 2005.

[18] M. Szilagyi, Electron and Ion Optics, Plenum Press: New York, 1988.

[19] P. W. Hawkes, Magnetic Electron Lenses, Springer-Verlag, Berlin, 1982.

[20] M. Kato, K. Tsuno, IEEE Transaction on Magnetic 26 (1990) 1023-1025.

[21] A. B. El-Kareh, J. C. J. El-Kareh, Electron Beams Lenses and Optics, vol.1 and 2, (Academic Press: New York and London), 1970.

[22] K. Tsuno, Y. Harada, J. Phys. E: Sci. Inst. 14 (1981a) 313-319.

[23] K. Tsuno, Y. Harada, J. Phys. E: Sci. Inst. 14 (1981b) 955-960. 